\title{
L'effet de l'usage du tableau blanc interactif (TBI) sur les pratiques pédagogiques dans trois écoles élémentaires de Strasbourg
}

\section{The effect of the use of the interactive whiteboard (IWB) on teaching practices in three elementary schools in Strasbourg}

\section{El efecto del uso de la pizarra digital interactiva (PDI) en las prácticas pedagógicas en tres escuelas de primaria de Estrasburgo}

Abdessamad Redouani, doctorant

Université de Strasbourg, France

abdessamad.redouani@etu.unistra.fr

RÉSUMÉ

Dans un contexte marqué par l'implantation des technologies éducatives dans les établissements scolaires en France et notamment l'équipement des écoles élémentaires par des tableaux blancs interactifs (TBI), l'objectif de ce travail de recherche est d'étudier l'effet de l'usage pédagogique de ce dispositif sur les pratiques enseignantes à l'école élémentaire. Ce sont sept enseignants, dans trois écoles élémentaires publiques de Strasbourg, qui ont fait l'objet d'un suivi et d'une étude pour cette recherche. Deux instruments de collecte de données ont été utilisés : des entretiens semi-directifs individuels et des observations directes en classe. Les résultats montrent que l'usage du TBI induit des transformations dans les pratiques pédagogiques des professeurs des écoles. Ces résultats montrent également que le TBI n'est pas utilisé à son plein potentiel et qu'une mise en place des formations spécifiques est perçue par les enseignants comme étant un facteur favorisant l'exploitation de toutes les fonctionnalités du TBI. 
Mots-clés : tableau blanc interactif (TBI), pratiques pédagogiques, dispositif techno-sémiopragmatique, interactivité

\section{ABSTRACT}

In a context marked by the implementation of educational technologies in schools in France and equipping elementary schools with interactive whiteboards (IWBs), this research aims to study the effect of this device's pedagogical use on teaching practices in elementary schools. Seven teachers in three public elementary schools in Strasbourg were monitored and observed for this research. Two data collection instruments were used: individual semistructured interviews and direct classroom observations. The results show that the use of IWB induces transformations in the teaching practices of school teachers. These results also show that the IWB is not used to its full potential and that teachers perceive that specific training is a factor favouring the exploitation of all the IWB's functionalities.

Keywords: interactive whiteboard (IWB), teaching practices, techno-semiotic-pragmatic device, interactivity

\section{RESUMEN}

En un contexto marcado por la implementación de tecnologías educativas en las escuelas de Francia y en particular por el equipamiento de las escuelas de primaria con pizarra digital interactiva (PDI), el objetivo de esta investigación es estudiar el efecto del uso educativo de este dispositivo en prácticas docentes en la escuela de primaria. Para esta investigación, se siguieron y estudiaron siete profesores en tres escuelas públicas de primaria en Estrasburgo. Se utilizaron dos instrumentos de recopilación de datos: entrevistas semiestructuradas individuales y observaciones directas en el aula. Los resultados muestran que el uso de PDI supone transformaciones en las prácticas docentes de los maestros de escuela. Estos resultados también muestran que el potencial de la PDI no suele utilizarse al completo y que la formación de capacitación específica es percibida por los maestros como un factor que favorece la explotación de todas las funcionalidades de la PDI.

Palabras clave: pizarra digítal interactiva (PDI), prácticas docentes, dispositivo tecno-semiopragmático, interactividad

\section{Introduction}

En France, comme dans plusieurs pays du monde, les salles de classe dans les établissements scolaires sont de plus en plus équipées de technologies dites « éducatives » telles que les ordinateurs, les tablettes tactiles..., mais aussi les tableaux blancs interactifs (TBI). Le TBI, appelé aussi TNI (tableau numérique interactif), est un tableau sur lequel il est possible d'afficher l'écran d'un ordinateur et de contrôler certaines de ses fonctions directement à l'aide d'un stylet ou avec le bout des doigts. II s'agit d'un dispositif qui « allie les avantages d'un écran tactile et de la vidéoprojection » (Trestini, 2018, p. 33). Récemment, l'apparition du VPI (vidéoprojecteur interactif) permet d'offrir les mêmes fonctions que celles du TBI avec la possibilité supplémentaire de projeter le contenu du cours sur un tableau blanc ordinaire. 
Le rapport établi par le député parlementaire Jean-Michel Fourgous (2010) considère la présence des TBI dans les établissements scolaires comme un indicateur primordial de modernisation de l'école. Rappelons que l'introduction des TBI dans les établissements scolaires français a commencé en 2004 avec l'opération PrimTICE (Fourgous, 2010). Cette opération a permis d'augmenter le nombre de TBI dans les écoles françaises en passant d'une dizaine à environ 2500 à la fin 2007 (Trestini, 2018). Ce processus ne cesse de continuer et le taux d'équipement double d'une année à l'autre. En 2018, selon les données issues de l'enquête ETIC, on compte environ 52151 TBI.

L'objectif de cette recherche est d'analyser les changements dans les pratiques enseignantes induites par l'usage du TBI dans trois écoles élémentaires publiques françaises. Autrement dit, nous nous posons la question de savoir si le TBI modifie la façon d'enseigner des enseignants.

\section{Revue de littérature : avantages éducatifs et limites du TBI}

Les différentes recherches portant sur le TBI rapportent que cet outil a des avantages pour l'enseignant et l'apprenant, malgré l'existence des limites liées à son utilisation. Ces recherches montrent que le TBI pourrait favoriser la pratique des pédagogies actives, mais qu'il n'est pas souvent utilisé de manière efficace (Lefebvre et Samson, 2015).

En effet, le TBI est un outil technologique connecté à l'Internet qui offre à l'enseignant plusieurs opportunités. Ainsi, il lui permet une meilleure présentation de contenus théoriques de façon multisensorielle (Saltan et Arslan, 2009). II lui permet aussi, contrairement au tableau classique, de sauvegarder du contenu pour le récupérer ultérieurement et de le partager ou de l'archiver (Dostal, 2011). Le TBI « permettrait d'activer des conflits sociocognitifs susceptibles de favoriser l'apprentissage » (Gilly, 1995, cité par Trestini, 2018, p. 33). En utilisant les fonctionnalités du TBI, les enseignants peuvent facilement interagir et communiquer avec les élèves et les engager davantage dans la leçon (Becta, 2003, cité par Karsenti, 2016).

Néanmoins, des chercheurs considèrent que les avantages du TBI restent potentiels ou supposés (Vertallier Monet, 2013). De fait, un nombre important d'études se sont basées sur les perceptions (ou les ressentis) des enseignants et des élèves obtenues à travers des questionnaires et des entretiens (Smith, Higgins, Wall et Miller, 2005). De plus, ces études n'ont pas prouvé qu'il puisse y avoir un impact direct du TBI sur la réussite scolaire (Karsenti, 2016).

Du côté de l'apprentissage, le principal avantage sur lequel s'entendent la plupart des chercheurs reste la motivation accrue des élèves (Boidou, 2019; Hesto, 2018). Cette motivation ne peut durer dans le temps que si elle est maintenue par des pratiques et des méthodes diversifiées de l'enseignant. Mais de quelle motivation parlons-nous? Est-ce de la motivation envers l'outil ou de la motivation vis-à-vis du contenu médié par l'outil? Si au début des années 2000 les élèves étaient attirés par l'effet de nouveauté des TBI ('’effet « wow »), les élèves d'aujourd'hui, désignés comme des « digital natives » (Prensky, 2001, cité par Dauve-Raeis, 2018) et donc habitués aux systèmes interactifs tels que les tablettes, les téléphones, etc., sont peut-être davantage attirés par la richesse des contenus d'enseignement.

Bien que les recherches montrent que le TBI présente un réel potentiel pédagogique, d'importantes limites liées à son utilisation ont été signalées. Les problèmes techniques et la rareté des ressources disponibles sont classés parmi les défis majeurs relatifs à l'usage du TBI en salle de classe. De plus, l'usage d'un tel 
outil est considéré comme chronophage pour les enseignants dont les responsabilités professionnelles sont déjà importantes (Manny-Ikan, Dagan, Tikochinski et Zorman, 2011).

\section{Cadre théorique}

\section{TBI, dispositif techno-sémio-pragmatique (Peraya,1999)}

Le concept de "dispositif " trouve ses origines dans le domaine technique où il désigne " un ensemble de moyens disposés conformément à un plan " (Charlier, Deschryver et Peraya, 2006). Ce concept est introduit au champ des sciences de l'éducation et de la formation depuis les années 1970. C'est « un ensemble de moyens humains et matériels agencés en vue de faciliter un processus d'apprentissage » (Blandin, 2002, cité par Charlier, Deschryver et Peraya, 2006, p. 470). Peraya $(1998,1999)$ a enrichi les travaux des chercheurs s'intéressant à l'analyse de ce concept en y intégrant les paramètres cognitifs liés à l'implication des acteurs (Bourdet et Leroux, 2009). II parle alors d'un dispositif techno-sémiopragmatique (TPS). C'est l'ensemble des interactions entre trois univers : une technologie, un système de relations, un cadre technosocial et un système de représentations de l'ordre du sémio-cognitif (Peraya, 1999). En ce sens, le TBI n'apparaît plus comme un outil isolé des autres outils présents dans la classe, mais comme un dispositif qui assure l'interaction entre des variables techniques, symboliques et humaines afin de favoriser la communication dans la salle de classe à travers des interactions susceptibles d'instaurer un apprentissage collaboratif.

\section{L'interactivité, principal attrait du TBI}

« La capacité d'une machine à réagir aux commandes et actions de son utilisateur est donc caractérisée par son interactivité » (Viallon et Trestini, 2019, p. 50). Linard (1996, cité par Petitgirard, Abry et Brodin, 2011) distingue la notion d'interactivité qui est d'ordre technique de celle de l'interaction qui est d'ordre humain. Selon Jacquinot et Meunier (1999, p. 1), « on peut parler de l'interactivité des machines et de I'interaction par la machine ». Les auteurs Éric Barchechath et Serge PoutsLajus distinguent interactivité fonctionnelle et interactivité intentionnelle (Julia, 2003). La première concerne l'échange d'information entre l'utilisateur et la machine, et la deuxième est liée à l'engagement de l'auteur face à l'utilisateur. Geneviève Jacquinot parle d'interactivité transitive, qu'elle oppose à l'interactivité intransitive (Julia, 2003). La première est celle qui gère le protocole de communication entre l'utilisateur et la machine, et la deuxième, celle qui gère le protocole de communication entre l'auteur et l'utilisateur. " L'auteur absent mais présent à travers le logiciel qu'il a conçu » (Petitgirard, Abry et Brodin, 2011, p. 38). L'interactivité rendue possible par le TBI a deux dimensions : interactivité technique et interactivité pédagogique (Smith, Higgins, Wall et Miller, 2005). Le TBI favorise le croisement entre ces deux types d'interactivité, par exemple lorsque la projection d'une vidéo ou des images oblige les élèves à s'interroger et à discuter (Beauchamp et Kennewell, 2008, cité par Alcheghri, 2016).

\section{Les pratiques pédagogiques}

Les pratiques pédagogiques désignent l'ensemble des actions de l'enseignant " consistant à mettre en place un certain nombre de conditions cognitives, matérielles, relationnelles, temporelles auxquelles les élèves sont confrontés " (Morlaix et Duguet, 2017, p. 128). Karsenti (1998) a montré que la pratique pédagogique d'un enseignant est composée des éléments observables (comportements et actions de l'enseignant) et des éléments non observables liés à la représentation de l'enseignement et de l'apprentissage propre à chaque enseignant. Ainsi, cet auteur définit la pratique pédagogique comme « le concept opératoire de l'agencement spécifique et personnel d'attitudes, d'activités et d'interventions 
particulières à chaque situation pédagogique, mais aussi le reflet de qualités personnelles de l'enseignant exprimées dans l'acte éducatif, avec le but de déclencher et de soutenir l'apprentissage des élèves » (Karsenti, 1998, cité par Karsenti, Savoie-Zajc et Larose, 2001, p. 94).

L'usage des technologies éducatives, et notamment celui du TBI (puisque c'est cet outil qui nous intéresse ici), a pour but de soutenir l'apprentissage des élèves. En effet, le TBI ne peut être utilisé que lorsqu'il apporte une plus-value pédagogique. II constitue un dispositif avec lequel l'enseignant peut transformer et renouveler ses pratiques en favorisant l'autonomie de l'apprenant dans la construction de ses apprentissages.

\section{MODĖLE D'ÉVALUATION DE L’USAGE DU TBI EN CLASSE : BEAUCHAMP (2004)}

Beauchamp (2004) propose un modèle comprenant cinq étapes de progression par lesquelles passent les enseignants lors de leur utilisation du TBI en classe.

1. Substitut au tableau noir : l'enseignant utilise le TBI comme un tableau traditionnel (pour écrire et dessiner).

2. Utilisateur apprenti : l'enseignant commence à utiliser d'autres logiciels que celui du TBI (PowerPoint) et des images, mais uniquement pour « décorer » ses documents.

3. Utilisateur initié : l'enseignant est conscient du potentiel du TBI pour changer et améliorer la pratique pédagogique.

4. Utilisateur avancé : " L'enseignant s'éloigne progressivement d'un enseignement linéaire et utilise des hyperliens, différents types de fichiers (image, sons, vidéos) dont certains numérisés (travaux d'élèves, leçons antérieures, pages de manuel scolaire) et de périphériques (caméra, tablette, microscope) pour bonifier son enseignement et faciliter la compréhension des contenus par les élèves » (Lefebvre et Samson, 2015, p. 42).

5. Utilisateur synergique : "L'enseignant et les élèves utilisent le plein potentiel du TBI de manière compétente, égale, fluide et intuitive pour Co construire des connaissances » (Lefebvre et Samson, 2015, p. 42).

\section{Problématique et hypothèses de recherche}

Dans cette étude, nous cherchons à évaluer si l'usage du TBI change les pratiques enseignantes à l'école élémentaire.

Des recherches antérieures stipulent que le TBI offre des fonctionnalités interactives capables de changer la pédagogie des enseignants (Lefebvre et Samson, 2015). Cependant, les TBI sont utilisés souvent comme de simples vidéoprojecteurs (Pacurar, 2015, Karsenti, 2016). Alors pourquoi ce phénomène?

Par ailleurs, la plupart de ces recherches n'ont pas prouvé qu'il puisse y avoir un impact positif de l'usage du TBI en classe sur la réussite scolaire (Karsenti, 2016). Pourtant, la volonté d'intégrer ces outils doit pouvoir être justifiée par un bénéfice sur le plan des résultats scolaires des élèves. N'y aurait-il pas des raisons particulières susceptibles d'expliquer ce phénomène que l'on pourrait qualifier de contre-productif au vu du coût que cette intégration massive implique? Une mauvaise utilisation de l'outil ou le peu d'engouement à utiliser toutes ses fonctionnalités seraient-ils à l'origine de ce résultat?

De ces différentes considérations, posons les hypothèses de recherche suivantes : 
1. L'usage du TBI engendre une transformation des pratiques pédagogiques chez les professeurs (utilisateurs de TBI) des écoles élémentaires. En effet, une enquête qualitative est nécessaire pour évaluer la part d'enseignants avant et après leur usage du TBI.

2. L'usage du TBI n'a que peu d'impact sur les résultats scolaires des élèves, car la plupart de ses fonctionnalités ne sont pas utilisées par l'enseignant.

3. La mise en place des formations relatives à l'utilisation des TBI est perçue par les enseignants comme étant un facteur favorisant l'exploitation des fonctionnalités du TBI.

\section{Méthode}

Notre méthodologie repose sur l'approche qualitative.

Les enquêtes de terrain portent sur trois écoles élémentaires publiques de Strasbourg en France. Ces écoles sont équipées des TBI depuis la rentrée scolaire 2016.

Pour réaliser l'enquête, deux outils de collecte de données ont été utilisés : des entretiens semi-directifs individuels auprès de sept enseignants et des observations directes en classe (trois observations). Un guide d'entretien a été établi sous forme de questions regroupées en plusieurs catégories. Pour les observations directes, une grille d'observation a été remplie lors de séances réalisées sans le TBI et d'autres avec un TBI. En plus, un mini-rapport a été rédigé pour chaque séance observée. Les données obtenues ont fait l'objet d'une analyse thématique. Enfin, il est à signaler que le critère du choix des participants à l'enquête est la volonté de participer exprimée par chaque enseignant.

\section{Résultats}

\section{La perception du TBI par les enseignants}

En répondant à la question : "Qu'est-ce que le TBI pour vous? », les enseignants interrogés perçoivent le TBI comme un outil pédagogique indispensable pour l'enseignement. Ils ont tous déclaré qu'ils ne pourraient plus s'en passer et revenir au tableau vert ou classique. De plus, ils y trouvent un outil qui facilite leur travail et les aide à mieux enseigner. Certains parlent d'un outil d'avenir, car ils pensent qu'avec le TBI l'école peut accompagner et suivre le développement que connait la société. Le tableau suivant résume les perceptions du TBI par les sept enseignants :

Tableau 1

Perception du TBI par les enseignants de notre échantillon

\begin{tabular}{|c|c|c|c|c|c|c|c|}
\hline Enseignant & $\mathbf{1}$ & $\mathbf{2}$ & $\mathbf{3}$ & $\mathbf{4}$ & $\mathbf{5}$ & $\mathbf{6}$ & $\mathbf{7}$ \\
\hline $\begin{array}{c}\text { Perception } \\
\text { du TBI }\end{array}$ & $\begin{array}{l}\text { Outil qui } \\
\text { remplace } \\
\text { les livres }\end{array}$ & $\begin{array}{l}\text { Outil principal } \\
\text { pour un } \\
\text { nouvel ensei- } \\
\text { gnement }\end{array}$ & $\begin{array}{l}\text { Outil de } \\
\text { l'avenir }\end{array}$ & $\begin{array}{l}\text { Outil qui } \\
\text { facilite les } \\
\text { appren- } \\
\text { tissages }\end{array}$ & $\begin{array}{l}\text { Un } \\
\text { médiateur }\end{array}$ & $\begin{array}{l}\text { Outil qui } \\
\text { permet de } \\
\text { montrer plein } \\
\text { de choses }\end{array}$ & $\begin{array}{l}\text { Outil qui } \\
\text { permet d'aug- } \\
\text { menter la } \\
\text { présentation }\end{array}$ \\
\hline
\end{tabular}




\section{L'usage du TBI par les enseignants}

Nous avons demandé aux enseignants quels étaient leurs principaux types d'usage du TBI en classe. Tous les enseignants interviewés ont déclaré qu'ils l'utilisent en permanence et pour enseigner toutes les matières scolaires : « Je l'utilise toute la journée. Il est allumé dès le matin à $8 \mathrm{~h}$ et je l'éteins le soir quand je pars » (enseignante 3).

Le principal logiciel utilisé par tous les enseignants sur le TBI est Workspace. Des enseignants ont déclaré qu'ils utilisent aussi les logiciels PowerPoint, Word, Excel et Publisher.

Voici les principaux usages du TBI déclarés par les enseignants :

- Insérer des documents, projeter des tableaux et des cartes : « En histoire c'est magique parce qu'on peut projeter des tableaux géants. Avant c'était en miniatures sur les livres. En géographie on peut projeter les cartes » (enseignante 2).

- Faire écouter des mots, des dialogues, des chants ou de la poésie : « En allemand je l'utilise beaucoup parce qu'on peut écouter les mots, les sons grâce à des petites "Simpsons" sur YouTube, chose que je ne pouvais pas faire avant » (enseignante 2 ).

- Chercher des documents et des définitions sur Internet (enseignante 2).

- Aider les apprenants à améliorer leur écriture : «On peut reproduire les lignes du cahier, on peut reproduire les mêmes couleurs qu'eux. On peut écrire dans toutes les polices qu'on veut, ce qu'on ne pouvait pas faire avant à la craie » (enseignante 3).

- Mettre des activités ludiques : « Je peux faire réviser mes élèves sur la table de multiplication grâce à des petits jeux » (enseignante 1).

D'après ces propos des enseignants, on peut constater que le TBI est utilisé la plupart du temps comme un support de projection et d'affichage. Ainsi, les enseignants se servent du TBI pour enrichir leurs leçons par des vidéos, des images, des cartes et des jeux interactifs. Cela pourrait être fait seulement à l'aide d'un simple vidéoprojecteur branché à l'ordinateur (moins coûteux). Or, les enseignants considèrent que le TBI ne peut pas être remplacé par un simple vidéoprojecteur : "Moi, je connais les vidéoprojecteurs avec les problèmes de câblage, brancher, apporter, dire aux enfants de faire attention à ne pas bouger le vidéoprojecteur donc sur le côté pratique c'est plus rapide le TBI » (enseignante 4).

L'utilisation des fonctionnalités du TBI diffère toutefois d'un enseignant à l'autre. Ainsi, certains enseignants pensent n'utiliser que $20 \%$ de ce qu'un TBI peut offrir, d'autres un peu plus. Mais aucun enseignant n'a déclaré qu'il utilise tout le potentiel du TBI, surtout que la dimension interactive du TBI est en pleine évolution, selon l'un des enseignants interviewés.

\section{L'impact du TBI sur les pratiques pédagogiques}

En répondant à la question : "Quel est l'impact du TBI sur vos pratiques pédagogiques? ", six enseignants sur sept disent que le fait d'avoir un TBI en classe change leurs pratiques enseignantes. "En termes de pratiques pédagogiques ça change beaucoup de choses, voire ça change tout » (enseignante 5); « Le TBI a changé ma façon d'enseigner » (enseignante 3).

Les transformations induites par l'usage du TBI dans les pratiques pédagogiques d'après ces enseignants sont :

- Faire évoluer la pratique pédagogique : «On peut augmenter nos leçons dans le sens numérique du terme. On peut très facilement jongler de la présentation à l'écrit à un document à une image qu'on va agrandir ou zoomer » (enseignant 7). 
- Varier les médias et les supports d'enseignement : «On peut partir d'une image, on peut partir d'un jeu, on peut partir d'une musique on peut partir d'une petite vidéo [...] pour lancer une séance " (enseignante 5).

- Enrichir le contenu des leçons : « Je vais pouvoir plutôt enrichir ma pratique grâce à cet outil [...] Je peux mettre plus de choses. Montrer plus de choses " (enseignante 1); « Dès qu'on a besoin d'un mot on a plus besoin de chercher sur le dictionnaire on va directement sur Internet » (enseignante 2).

- Faciliter le travail : « Ça facilite un peu la vie de l'enseignant en fait » (enseignante 5).

- Favoriser l'apprentissage par le jeu : «Ça nous permet aussi de plus facilement faire entrer le jeu d'ailleurs dans les apprentissages. L'apprentissage par le jeu est beaucoup plus facile avec le TBI que sans » (enseignante 5).

\section{La plus-value pédagogique du TBI}

En répondant à la question : «Qu'est-ce que le TBI a apporté depuis son installation dans votre classe en termes de plus-value pédagogique? », les enseignants ont déclaré que le TBI :

- Permet de gagner du temps : «Maintenant je suis moins stressée. J'ai plus du temps pour m'occuper d'autres choses » (enseignante 3); « Ça nous permet d'être beaucoup plus rapides, donc ça correspond beaucoup plus à la société d'aujourd'hui » (enseignant 5).

- Permet une meilleure présentation des contenus des leçons : "La possibilité de montrer d'autres choses, de projeter plein de choses » (enseignante 1); " Je peux montrer d'une meilleure façon les choses » (enseignante 3).

- Augmente la motivation, l'attention et la participation des apprenants : "ça les stimule beaucoup » (Enseignante 6); « Ils ont envie d'aller au tableau. Et quand je dis, qui veut aller au tableau? J'ai 26 doigts sur 26, ils veulent tous y aller parce que le fait de toucher que ce soit lumineux qu'on puisse utiliser un stylet ou le doigt ils adorent et même de projeter des images en fait j'arrive à capter l'attention en montrant des images en écoutant de la musique. Même les enfants qui ont des difficultés puisque c'est devenu un jeu ils veulent y aller. Du coup rares sont les enfants qui n'aiment pas y aller » (enseignante 1).

- Augmente le degré des interactions dans la classe : "L'interaction entre les élèves est vive et intéressante et entre moi et eux aussi. Et cela donne de la vie en fait à la classe [...] ça crée une communication dans la classe qui n'est pas forcement avec le tableau classique [...] le TBI crée une sorte d'ambiance du travail dans la classe » (enseignante 1).

- Est un médiateur : «C'est un outil qui nous permet de montrer de l'information ou de récupérer de l'information » (enseignant 5).

- Développe des compétences transversales chez les apprenants; « Le TBI permet aussi aux apprenants de développer des compétences en informatique » (enseignante 3).

- Permet de sauvegarder les leçons : «La mémoire du TBI ».

Les enseignants s'accordent sur le fait que le TBI apporte avant tout un gain de temps et une meilleure présentation des contenus théoriques, ce qui permet d'augmenter la motivation et l'attention des élèves. De plus, selon l'un de ces enseignants, le TBI permet d'augmenter le degré des interactions enseignantélèves et élèves-élèves. 


\section{L'impact du TBI sur les résultats scolaires des élèves}

Nous avons demandé aux enseignants quel était l'impact de l'utilisation du TBI en classe sur les résultats scolaires des élèves. Les réponses diffèrent d'un enseignant à l'autre. Ainsi, l'enseignante 2 pense que si la concentration et la motivation sont meilleures et si les élèves sont plus actifs alors, par conséquent, ils auront de meilleurs résultats. Pour l'enseignante 3 , le fait de gagner du temps grâce au TBI peut aider à avoir de meilleurs résultats, surtout pour les élèves en difficulté en leur consacrant plus du temps.

Pour les autres enseignants, le TBI n'est pas perçu comme un vecteur qui améliore les résultats scolaires des élèves :

« Mais je ne pense pas qu'il y a un lien de cause à effet direct entre le TBI et les résultats. II y a des élèves qui sont en très grande difficulté, pourtant ils ont un TBI. D'autres qui n'avaient pas de TBI dans une autre école sont très bons » (enseignante 6).

\section{Les formations des enseignants à l'usage du TBI}

Parmi les sept enseignants interviewés, quatre ont déclaré avoir bénéficié d'au moins une formation relative à l'usage du TBI, alors que trois n'ont pas eu de formations. Cependant, seulement une enseignante parmi les quatre ayant bénéficié des formations a montré sa satisfaction vis-à-vis de ces formations en disant : "Oui, suffisamment, ça m'a suffi. Après, les informations, on peut les trouver par soi-même. Oui si j'estime avoir été bien formée, je dis oui » (enseignante 5).

En revanche, les autres enseignantes formées pensent que les formations qu'elles ont eues ne leur permettent pas d'exploiter tout le potentiel du TBI : « On a eu des formations assez courtes. Ça veut dire que si on avait plus de formations, on pourrait utiliser d'autres fonctionnalités que je ne connais pas encore. Parce qu'il y a plein de choses que je ne connais pas et je les découvre des fois par hasard, mais effectivement en formation si on en avait un peu plus ça serait bien, on pourrait utiliser plein de choses » (enseignante 1).

Nous pouvons donc constater que l'équipement des écoles par les TBI n'a pas été accompagné par des formations suffisantes pour les enseignants. De plus, la plupart des enseignants attendent la mise en place des formations par l'académie pour pouvoir améliorer leurs compétences relatives à l'usage des fonctionnalités du TBI. L'autoformation est limitée selon les enseignants par la contrainte temporelle.

\section{Limites et défis du TBI}

À la fin de chaque entretien auprès des enseignants, nous avons abordé la question des limites relatives à l'usage du TBI. Les enseignants interrogés ont indiqué rencontrer des problèmes techniques dans l'utilisation du TBI en classe. Le manque de formation est aussi évoqué parmi ces limites. L'autre inconvénient souligné concerne l'inquiétude de ces enseignants par rapport aux effets négatifs des écrans sur la vision des enfants.

Pour ces enseignants, les défis de TBI seraient :

- De procéder à des améliorations techniques de l'objet : «Peut-être améliorer la précision du stylet. Lorsque j'écris, c'est moins joli que lorsque j'écrivais sur un tableau blanc normal avec la craie » (enseignante 6).

- D'être plus formés pour « arriver à tout utiliser et de manière régulière » (enseignante 3). 


\section{Discussion}

Les hypothèses émises par notre recherche ont été validées.

En effet, nous avons fait l'hypothèse d'une transformation des pratiques pédagogiques des enseignants à la suite de l'usage du TBI. Les données obtenues par entretiens et observations nous ont permis de valider notre première hypothèse. Ainsi, les enseignants interviewés pensent que le TBI change leur façon d'enseigner, comme il leur permet de mieux présenter les activités d'apprentissages, de varier les méthodes et d'enrichir les leçons via les différents outils audiovisuels utilisés ainsi que l'accès à Internet qu'il permet. De plus, ces enseignants nous ont confirmé que le TBI facilite leur travail, leur permet de gagner du temps et de sauvegarder tout ce qu'ils ont fait.

S'agissant de l'utilisation des fonctionnalités du TBI, les pratiques déclarées par les enseignants de notre échantillon ainsi que celles observées ont été analysées en fonction du modèle de Beauchamp (2004). D'après cette analyse, nous constatons que ces enseignants développent des pratiques du TBI qui peuvent être associées aux quatre premiers stades de ce modèle (voir le modèle de Beauchamp dans le cadre théorique) sans pour autant atteindre le stade d' "utilisateur synergique ». Ainsi, d'après le modèle de Beauchamp (2004), un enseignant est considéré comme utilisateur synergique lorsqu'il utilise avec ses élèves le plein potentiel du TBI de manière compétente, égale, fluide et intuitive pour coconstruire des connaissances (Lefebvre et Samson, 2015).

Le faible usage du potentiel du TBI n'a pas permis d'observer un impact direct et significatif de l'usage de ce dispositif sur les résultats scolaires des élèves (hypothèse 2). Les facteurs qui limitent l'exploitation de toutes les fonctionnalités du TBI, selon les résultats de notre étude, sont le manque de formation et d'accompagnement, mais aussi une faible initiative prise par les enseignants eux-mêmes. Les résultats de notre étude révèlent qu'une formation et un accompagnement pédagogique sont considérés par les enseignants comme une nécessité pour une meilleure utilisation du TBI (hypothèse 3 ). Ainsi, les enseignants ont besoin de sessions de formation sur l'usage du TBI afin de mieux intégrer des activités interactives plus centrées sur l'apprenant.

\section{Conclusion}

Cette étude nous a permis de faire le point sur les transformations pédagogiques induites par l'usage du TBI à l'école élémentaire. Accès à Internet, augmentation de la présentation des contenus, enrichissement de l'apprentissage par le jeu et augmentation du degré des interactions en classe ne sont que quelquesuns des apports du TBI sur les pratiques pédagogiques citées. Malgré que les enseignants de notre échantillon ont déclaré que le TBI leur permet de modifier leurs pratiques quotidiennes, nous avons constaté, d'après leurs pratiques déclarées et observées, qu'ils l'utilisent dans la plupart du temps comme un outil de présentation.

La taille réduite de notre échantillon (sept enseignants) et les outils de recherches choisis ne nous ont pas permis de mieux montrer l'intérêt de l'introduction de cet outil, ce que peut constituer une limite à notre recherche. Nous envisageons donc de réaliser une recherche plus large avec une méthodologie mixte (quantitative et qualitative) auprès des enseignants usagers et non usagers du TBI pour mieux évaluer l'influence de ce dispositif sur l'enseignement et l'apprentissage. 


\section{Liste de références}

Alcheghri, H. (2016). Usages pédagogiques du tableau numérique interactif (thèse de doctorat, Université Lumière Lyon 2 , Lyon, France).

Beauchamp, G. (2004). Teacher use of the interactive whiteboard in primary schools: towards an effective transition framework, Technology, Pedagogy and Education, 13(3), pp. 327-348, University of Wales Swansea, United Kingdom.

Boidou, B. N. (2019). Facteurs d'influence de l'impact d'un usage partagé du tableau blanc interactif sur la performance scolaire dans un établissement d'enseignement secondaire général de Côte d'lvoire. Thèse de doctorat, Education. Université de Cergy Pontoise, 2019. Français. ffNNT : 2019CERG1003ff. fftel-02524668.

Bourdet, J. F. et Leroux, P. (2009). Dispositifs de formation en ligne : de leur analyse à leur appropriation, Distance et savoirs, vol. $7 \mathrm{n}^{\circ} 1$, p. 11-29, Laboratoire d'Informatique de l'Université du Maine.

Charlier, B., Deschryver, N. et Peraya, D. (2006). Apprendre en présence et à distance : Une définition des dispositifs hybrides. Distances et savoirs, vol. 4(4), 469-496. https://www.cairn.info/revue-distances-et-savoirs-2006-4-page469.htm.

Dauve-Raeis, V. E. C. (2018). Étude de cas sur l'intégration des tablettes et du tableau blanc interactif dans un établissement primaire genevois: facteurs d'appropriation chez les enseignants (Doctoral dissertation, University of Geneva).

Dostal, J. (2011). Reflections on the use of interactive whiteboards in instruction in international context. The New Educational Review.

Fourgous, J. M. (2010). Réussir l'école numérique. Rapport de la mission parlementaire sur la modernisation de l'école par le numérique. Paris : Mission parlementaire de Jean-Michel Fourgous sur l'école numérique.

Hesto, J. (2018). L'impact du TBI sur la motivation et la réussite scolaire chez les élèves. Education. dumas-01938967.

Jacquinot, G. et Meunier, C. (1999). Introduction. L'interactivité au service de l'apprentissage. Revue des sciences de l'éducation.

Julia, J. T. (2003). Interactivité, modes d'emploi. Réflexions préliminaires à la notion de document interactif. Documentaliste-Sciences de l'Information, (vol. 40), p. 204-212.

Karsenti, T. (1998). Étude de l'interaction entre les pratiques pédagogiques d'enseignants du primaire et la motivation de leurs élèves, thèse de doctorat présentée à l'Université du Québec à Montréal.

Karsenti, T. (2016). Le tableau blanc interactif (TBI) : usages, avantages et défis. Montréal : CRIFPE.

Karsenti, T., Savoie-Zajc, L. et Larose, F. (2001). Les futurs enseignants confrontés aux TIC : changements dans l'attitude, la motivation et les pratiques pédagogiques. Éducation et Francophonie.

Lefebvre, S. et Samson, G. (2015). Le tableau numérique interactif : Quand chercheurs et praticiens s'unissent pour dégager des pistes d'action. Presses de l'université de Québec.

Manny-lkan, E., Dagan, O., Tikochinski, T. B. et Zorman, R. (2011). Using the interactive white board in teaching and learning- An evaluation of the SMART CLASSROOM pilot project. Interdisciplinary Journal of E-Learning and Learning Objects, 7, 249-273.

Morlaix, S. et Duguet, A. (2017). Les pratiques pédagogiques des enseignants du secondaire: l'éclairage des comparaisons internationales. Éducation comparée. Nouvelle série (halshs-01521724).

Pacurar, E. (2015). L'appropriation du tableau numérique interactif et du manuel numérique chez des enseignants du second degré : valeurs ressources et affordances contextuelles, revue La Recherche en éducation, AFIRSE, ISSN 1647-0117, nr. 13.

Peraya, D. (1998). Théories de la communication et technologies de l'information et de la communication. Un apport réciproque. Revue européenne des sciences sociales, vol. XXXVI, n²111, 1998, p. 171-188.

Peraya, D. (1999). Médiation et médiatisation : le campus virtuel. Hermès, La Revue, 25, 153167. https://doi.org/10.4267/2042/14983

Petitgirard J.Y., Abry D. et Brodin E. (2011). Le tableau blanc interactif. Paris : CLE international.

Saltan, F. et Arslan, K. (2009). A new teacher tool, interactive white boards : A meta-analysis. 
Smith, H. J., Higgins, S., Wall, K. et Miller, J. (2005). Interactive whiteboards: Boon or bandwagon? A critical review of the literature. Journal of Computer Assisted Learning, 21(2), 91-101.

Trestini, M. (2018). La modélisation d'environnements numériques d'apprentissage de nouvelle génération. Londres : ISTE Editions.

Vertallier Monet, S. (2013). L'utilisation du TBI en classe de FLE ou comment susciter des interactions orales. Sciences de l'Homme et Société. dumas-00839480.

Viallon, P. et Trestini, M. (2019). Cultures numériques : cultures paradoxales? Collection Communication et Civilisation. Paris : L'Harmattan. 